\title{
Circ_0004913 sponges miR-1290 and regulates FOXC1 to inhibit the proliferation of hepatocellular carcinoma
}

\author{
Yabin Yu', Suyang Han², Meng Li ${ }^{3}$, Yan Song ${ }^{1}$ and Fuzhen Qi ${ }^{1 *}$ (D)
}

\begin{abstract}
Background: Circular RNA (circRNA), an novel type of non-coding RNA, could interact with miRNA and protein molecules to regulate the occurrence and progression of hepatocellular carcinoma (HCC). However, little is known about the pathogenesis of circ_0004913 in HCC.

Materials: Through the GEO (Gene Expression Omnibus database) to find dysfunctional circRNAs in HCC, and circ_0004913 was selected as the research object. Quantitative reverse transcription PCR (qRT-PCR) was used to detect the expression level of circ_0067934 in HCC tissues and cells. CCK-8, Edu and flow cytometry assays were used to determine the malignant behavior of transfected HCC cells. Mechanistically, RNA immunoprecipitation and dualluciferase reporter gene assay were performed to explore the relation between circ_0067934, miR-1290 and FOXC1 (Forkhead box C1) in HCC.
\end{abstract}

Results: The expression of circ_0004913 was down-regulated in HCC tissues and cell lines, while the overexpression of circ_0004913 attenuates the malignant behavior of HCC cells. Bioinformatics predicted that circ_0004913 interacts with miR-1290, which targeted FOXC1 mRNA. In fact, miR-1290 promoted the malignant behavior of HCC cells, while FOXC1 had the opposite effect. In addition, circ_0004913 overexpression enhanced FOXC1 expression by reducing miR-1290 expression, thereby inhibiting the proliferation of HCC cells.

Conclusions: Circ_0004913 / miR-1290 / FOXC1 regulatory axis could inhibit the progress of HCC. Our findings may provide potential new targets for the diagnosis and treatment of HCC.

Keywords: Circ_0004913, miR-1290, FOXC1, Proliferation, Hepatocellular carcinoma

\section{Introduction}

Accounting for approximately $90 \%$ of primary liver cancer, Hepatocellular carcinoma (HCC) is the third leading cause of cancer-related deaths in the world $[1,2]$. Chronic hepatitis $B$ virus (HBV), hepatitis $\mathrm{C}$ virus (HCV), heavy alcohol consumption and diabetes are the main risk factors for HCC [3]. There are currently several treatments

\footnotetext{
${ }^{*}$ Correspondence: qifuzhen2017@163.com

${ }^{1}$ Department of Hepatobiliary Surgery, The Affiliated Huaian No.1

People's Hospital of Nanjing Medical University, 1 West Huanghe Road, Huai'an 223300, Jiangsu, People's Republic of China

Full list of author information is available at the end of the article
}

for HCC, including surgical resection, chemoradiation, and liver transplantation. Sometimes, hepatocellular carcinoma requires a multidisciplinary treatment to get the best results [4]. However, the 5-year overall survival rates of patients with $\mathrm{HCC}$ remain low, largely because of metastasis and recurrence [5]. In order to improve the diagnosis and prognosis of HCC patients, it is particularly critical to discover and identify new targets for precise treatment.

Circular RNAs (circRNAs) are a new type of non-coding RNAs, characterized by continuous covalent closed loop without $5^{\prime}$-cap structure or $3^{\prime}$-poly A tail, and considered as a by-product of splicing error [6]. Aberrant

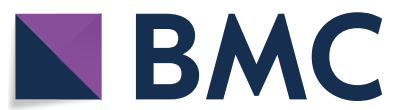

(c) The Author(s) 2020. This article is licensed under a Creative Commons Attribution 4.0 International License, which permits use, sharing, adaptation, distribution and reproduction in any medium or format, as long as you give appropriate credit to the original author(s) and the source, provide a link to the Creative Commons licence, and indicate if changes were made. The images or other third party material in this article are included in the article's Creative Commons licence, unless indicated otherwise in a credit line to the material. If material is not included in the article's Creative Commons licence and your intended use is not permitted by statutory regulation or exceeds the permitted use, you will need to obtain permission directly from the copyright holder. To view a copy of this licence, visit http://creativeco mmons.org/licenses/by/4.0/. The Creative Commons Public Domain Dedication waiver (http://creativecommons.org/publicdomain/ zero/1.0/) applies to the data made available in this article, unless otherwise stated in a credit line to the data. 
expression of circRNAs have also been shown to be associated with the initiation and development of various diseases, including cancers [7]. For example, circRNA FARSA promoted colorectal cancer cell growth, while circRNA UBAP2 increased SEMA6D expression to enhance cisplatin resistance in osteosarcoma $[8,9]$. Various circRNAs exist in mammalian cells and regulate a broad range of biological processes through various mechanisms, including sponging of microRNAs (miRNAs) [10]. CircRNA circ_0054537 could sponge miR-130a-3p to promote the progression of renal cell carcinoma through regulating cMet pathway, and circ_0008532 promotes bladder cancer progression by regulation of the miR155-5p/miR-330-5p/MTGR1 axis [11, 12]. However, the role of circ_0004913 in cancer is poorly understood.

Based on the information in the circbase database, circ_0004913, encoded by the TEX2(Testis expressed 2) gene, is located on chromosome chr17: 62,248,459$62,265,775$ and about 495 bp [13]. In the HCC microarray data of GEO, the down-regulated circ_0004913 was selected as the research object. We hypothesized that circ_0004913 is involved in the malignant process of $\mathrm{HCC}$ and conducted a series of in vitro proliferationrelated experiments. Circ_0004913 could significantly inhibit the proliferation of HCC cells in vitro and arrest more HCC cells in G1 phase. Circ_0004913 could also up-regulate the expression of FOXC1 (Forkhead box C1) in HCC cell line by interacting with miR-1290. These findings provide a new perspective into the function of circ_0004913 and highlight the potential of developing HCC treatments.

\section{Materials and methods \\ Microarray analysis}

A comprehensive database of gene expression (GEO, http://www.ncbi.nlm.nih.gov/geo), which is a public repository for archiving and distributing microarrays for free, was screened. GSE97332 and GSE94508 were selected, and the "limma" package was used to analyze differences in gene expression between tumor and nontumor tissues $[14,15]$. CircRNAs with adjusted $\mathrm{P}<.05$ and $|\log \mathrm{FC}|>1$ were considered as significant dysregulated circRNAs.

\section{Patients}

A total of $50 \mathrm{HCC}$ patients who underwent surgical treatment in our hospital were enrolled from June 2012 to June 2015. The main screening criteria include: (1) histological diagnosis of primary HCC; (2) receiving surgical resection; (3) not receiving chemotherapy or radiotherapy before surgery; (4) complete preoperative tumor characteristics. In addition, this study Patients with relapsed or secondary HCC and a history of malignant tumors were excluded. The study was approved by the hospital's ethics committee. All patients or their families provided written informed consent.

\section{Cell lines}

Human HCC cell lines Hep3B, HepG2, SMMC-7721, and Huh-7, and the human normal liver cell line (LO2) were purchased from the Chinese Academy of Sciences (Shanghai, China) and were cultured in Dulbecco's modified Eagle's medium (DMEM; Gibco, California, USA) supplemented with $10 \%$ fetal bovine serum (Gibco) and antibiotics $(100 \mathrm{U} / \mathrm{ml}$ penicillin $\mathrm{G}$ and $100 \mathrm{mg} / \mathrm{ml}$ streptomycin) at $37{ }^{\circ} \mathrm{C}$ in a humidified atmosphere with $5 \%$ $\mathrm{CO}_{2}$.

\section{QRT-PCR}

Total RNA was isolated with TRIzol Reagent ((Beyotime, Shanghai, China)) following the manufacturer's instruction. Then, $1 \mu \mathrm{g}$ total RNA was reversed into $20 \mu \mathrm{l}$ complementary DNA (cDNA) with First Strand cDNA Synthesis Kit (Takara, Tokyo, Japan). QRT-PCR was conducted using SYBR Green Master Mix II (Takara) on ABI7900 system (Applied Biosystems, CA, USA) in line with the manufacturer's procedure. Circ_0004913, miR1290, TEX2 and FOXC1 mRNA expression was determined using the $2^{-\Delta \Delta C T}$ method. A P-value $<0.05$ denotes a statistical significance. GAPDH and U6 were used as internal controls for circRNA and miRNA, respectively. All primers were purchased from GenePharma (Shanghai, China). The primer sequences are as follows: Circ_0004913: Forward: 5'-TACGTTGATCACCAA GGGCT-3', Reverse: 5'-CTTCTGCTTTGGCTGTGA CA-3'; miR-1290: Forward: ACACTCCAGCTGGGT GGATTTTTGGATC, Reverse: CTCAACTGGTGTCGT GGAGTCGGCAATT; FOXC1: Forward: 5'-AAAAAA TTGGAGGCTGCTT-3', Reverse: 5'-CCAAAGAAA AATCCCCACA-3'; GAPDH: Forward: 5'-AAGGTG AAGGTCGGAGTCA-3', Reverse: 5'-GGAAGATGG TGATGGGATTT-3'; U6: Forward: 5'-CTCGCTTCG GCAGCACATATACT-3', Reverse: 5'-ACGCTTCAC GAATTTGCGTGTC-3'.

\section{RNase R treatment assay}

Two $\mu \mathrm{g}$ RNA and 6 units of RNase R (Geneseed Biotech, Guangzhou, China) were added together to incubate for $20 \mathrm{~min}$ at $37^{\circ} \mathrm{C}$. Subsequently, circ_0004913 and TEX2 mRNA expression was detected through qRT-PCR.

\section{Subcellular localization}

The subcellular localization of circ_0004913 was detected using the PARIS Kit (Invitrogen, CA, USA) in accordance with the manufacturer's instructions. 


\section{Transfection}

The lentivirus-pHBLV-CMV-Cicr-MCS-EF1-circ_0004913, lentivirus-microRNA-1290 mimics, and their negative control were purchased from GenePharma. HCC cells were transduced with individual types of lentivirus at a multiplicity of infection (MOI) of 10 in the presence of $5 \mu \mathrm{g} / \mathrm{ml}$ puromycin (Thermo Fisher).

\section{CCK-8 assay}

Transfected SMCC-7721 and Huh-7 cells (1000 cells/ well) were seed onto 96-well plates and incubated overnight at $37{ }^{\circ} \mathrm{C}$. Cell proliferation was measured using the Cell Counting Kit-8 (CCK8, Dojindo, Shanghai, China) according to the manufacturer's protocol. OD values were measured at a wavelength of $450 \mathrm{~nm}$ using a microplate reader (Bio-Rad, CA, USA).

\section{5-Ethynyl-2'-deoxyuridine (Edu) assay}

The EdU proliferation assay (RiboBio, Guangzhou, China) was carried out according to the manufacturer's instructions. Transfected cells were incubated with $50 \mu \mathrm{M}$ EdU for $2 \mathrm{~h}$. Then an Apollo staining and DAPI staining were performed according to the instructions to detect the EdU positive cells with a fluorescence microscope.

\section{Flow cytometric analysis}

Transfected cells were suspended in $70 \%$ cold ethanol overnight after harvest. Then, cells stained with propidium iodide (PI) (Vazyme, Nanjing, China) for $30 \mathrm{~min}$ were analyzed. The proportion of cells in different cycle phases were calculated and compared.

\section{Luciferase assay}

Dual luciferase reporter system psiCHECKTM (Thermo Fisher) was used for luciferase assay. The wild type (wt) sequences and its mutant type (mut) sequences were cloned into the plasmid psiCHECK2. HEK293T cells $\left(5 \times 10^{4}\right.$ cells/well $)$ were cultured in 24-well plates overnight and transfected with $400 \mathrm{ng}$ of psiCHECK vector (psiCHECK-circ_0004913 wt, psiCHECK-circ_0004913 mut, or psiCHECK-FOXC1 wt, psiCHECK-FOXC1 mut), together with the plasmid for Renilla luciferase expression by lipofectamine 3000 . One day later, the luciferase assays were performed after co-transfection with miR-1290 mimics or NC.

\section{RNA immunoprecipitation (RIP) assay}

The EZMagna RIP kit (Merck, Darmstadt, Germany) was employed for RIP assay according to the manufacturer's protocol. In brief, RIP lysis buffer was used to HEK293T cells, and the lysate products were incubated at $4{ }^{\circ} \mathrm{C}$ for $6 \mathrm{~h}$ with magnetic beads that were pre-conjugated with anti-Argonaute 2 (AGO2) or anti-IgG antibody. Afterwards, the beads were washed and digested with protease $\mathrm{K}$, so as to remove the proteins. At last, the purified RNA was analyzed by qRT-PCR.

\section{Western blot}

Transfected cells were lysed in ice-cold RIPA buffer (Beyotime) with $10 \mathrm{nM}$ PMSF for $30 \mathrm{~min}$ and then collected to extract total protein. Total Proteins lysates were fractionated by sodium dodecyl sulfate polyacrylamide gel electrophoresis (SDS-PAGE) and then were transferred to polyvinylidene fluoride (PVDF) membrane. The membrane was blocked in 5\% non-fat milk in TBST for $2 \mathrm{~h}$ at room temperature and then immunostained overnight at $4^{\circ} \mathrm{C}$ using rabbit anti-FOXC1 and PCNA (1:1000, Cell Signaling Technology, CST, USA). Rabbit anti-GAPDH (CST) was taken as a control. The signals were captured and the intensity of the bands was quantified by using the ChemiDoc XRS + system (Bio-Rad).

\section{Mice xenograft models}

For animal experiment, male BALB/c nude mice (4-6-week-old) were bought from Nanjing Medical University (Nanjing, China) and randomly divided into 2 groups ( $\mathrm{n}=5$ per group). A total of $1 \times 10^{6}$ SMCC-7721 cells transfected with circ_0004913 or con were subcutaneously injected into mice. The tumor volume was calculated every week by the formula: length $\times$ width $^{2} / 2$ method. After 5 weeks, the tumors were removed for subsequent experiments. This study was approved by the Animal Committee of affiliated Huaian No.1 People's Hospital of Nanjing Medical University.

\section{Statistical analysis}

Data were shown as Mean \pm SD performed at least three independent replicates. SPSS software, 24.0 (SPSS Inc., Chicago, IL, USA) and Graphpad Prism 7.0 (San Diego, CA, USA) were used for one-way ANOVA (multiple groups), a two-tailed Student t-test (2 groups). KaplanMeier method test were used for survival analysis. Differences were considered as statistically significant if $\mathrm{P}<.0 .05$.

\section{Results}

The expression of circ_0004913 was downregulated in HCC tissues and cells

In order to study the potential role of circRNAs in the regulation of HCC process, GSE97332 and GSE94508 were selected from GEO for microarray analysis, and the expression profiles of circRNA in HCC tissues and nontumor tissue were compared. As shown in Fig. 1a, compared with non-tumor tissues, there are 82 differentially 


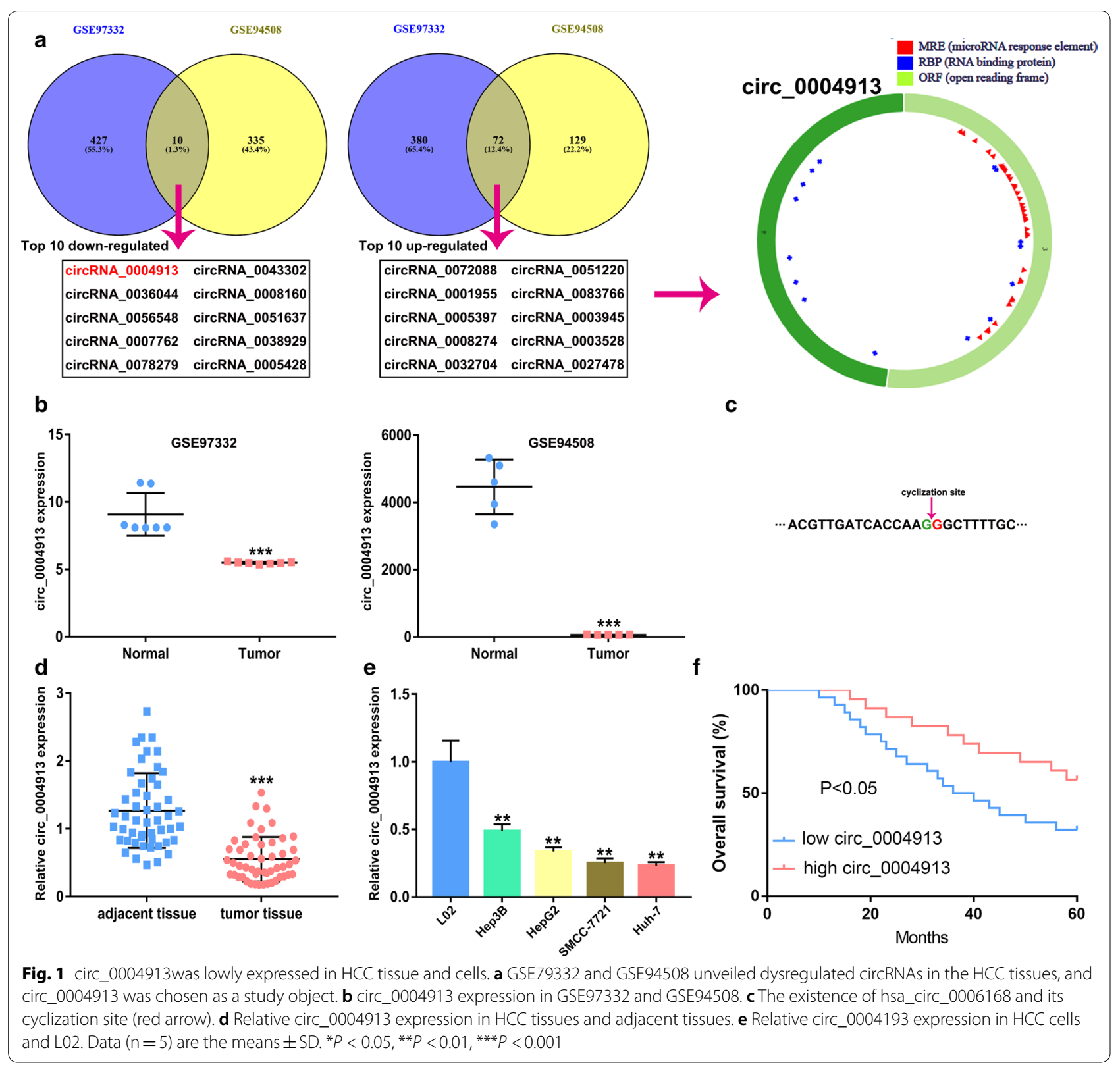

expressed circRNAs in HCC tissues, 72 of which were up-regulated and 10 were down-regulated. According to the values of the $\operatorname{LogFC}$, the top 10 dysregulated circRNAs were shown. Therefore, circ_0004913 was selected as the research object. circ_0004913, encoded by the TEX2 gene, is located on chromosome chr17: $62,248,459-62,265,775$ and about 495 bp. It was significantly reduced in the HCC tissues selected from GEO, compared with the normal tissues (Fig. 1b). Studies have confirmed the sequence of the cleavage point of the cyclization site in circ_0004913 [16] (Fig. 1c). Further analysis showed that the relative level of circ_0004913 expression in $50 \mathrm{HCC}$ tissues was significantly lower than that in the adjacent liver tissues (Fig. 1d). Similarly, the relative level of circ_0004913 expression in HCC cells was significantly lower than that in L02 cells (Fig. 1e).

To further evaluate the relationship of circ_0004913 and clinical pathological features, we divided the HCC tissues into two group with circ_0004913 high expression or low expression according to circ_0004913 median expression valve. Stratified analysis showed that the lower expression of circ_0004913 was significantly associated with larger tumor size, vascular invasion, advanced TNM stage and Edmondson grade, but no other tests 

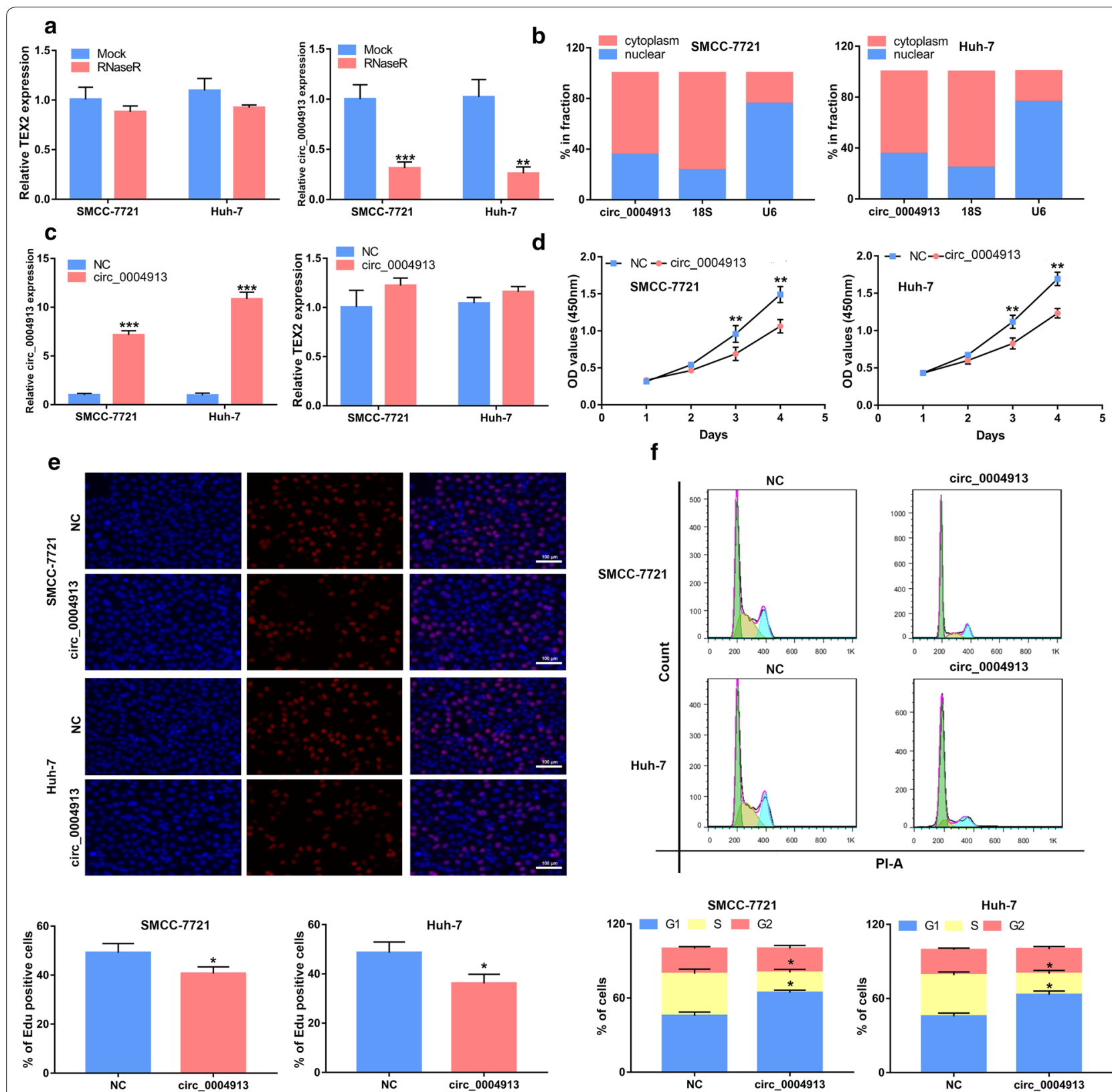

Fig. 2 circ_0004913 affected proliferation and cell cycle of HCC cells. a After RNase R treatment, circ_0004913 expression level and TEX2 mRNA expression level in SMCC-7721 and Huh-7 cells were measured. b Nuclear and cytoplasmic mRNA fraction experiment displays the location of circ_0004913 in SMCC-7721 and Huh-7 cells. c The expression levels of circ_0004913 and TEX2 in transfected SMCC-7721 and Huh-7 cells. d The proliferation capacities of transfected SMCC-7721 and Huh-7 cells were detected by CCK-8 assays. e The proliferation capacities of transfected SMCC-7721 and Huh-7 cells were detected by Edu assays. f Flow cytometry analysis revealed the cycle arrest of transfected SMCC-7721 and Huh-7 cells. Data $(\mathrm{n}=5)$ are the means \pm SD. ${ }^{*} P<0.05,{ }^{* *} P<0.01,{ }^{* *} P<0.001$

were performed on this population (Table 1). KaplanMeier analysis revealed that low circ_0004913 expression also was positively associated with poor overall survival of HCC patients (Fig. 1f). Therefore, the down-regulated circ_0004913 expression may be associated with the occurrence and progression in HCC.
Circ_0004913 overexpression suppressed the proliferation of HCC cells

Total RNA was extracted from SMCC-7721 and Huh-7 cells treated with RNase R. The expression level of linear TEX2 mRNA was significantly reduced, while the expression level of circ_0004913 did not change significantly. 


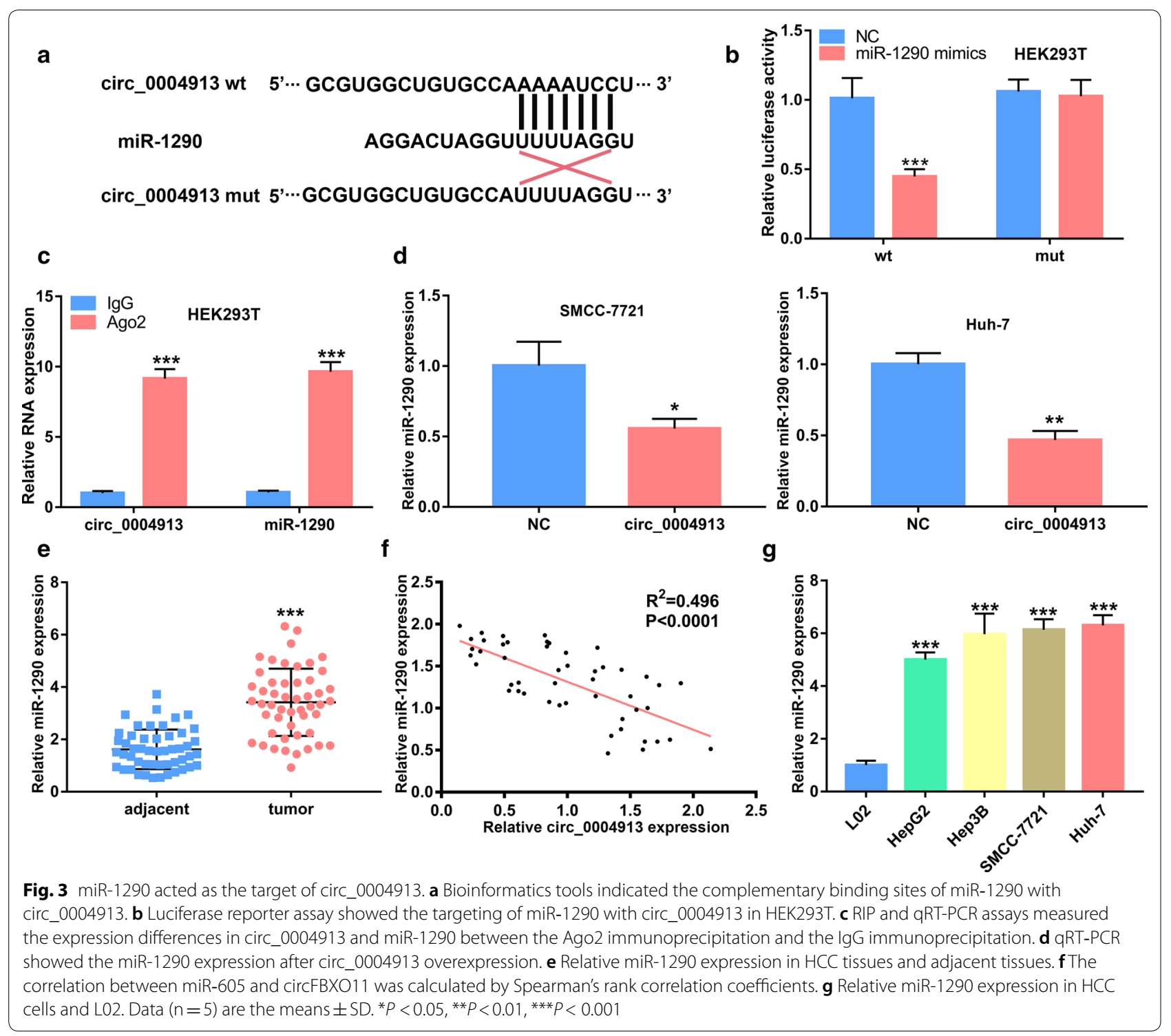

Thus, circ_0004913 was proved to be more stable than its linear TEX2 mRNA (Fig. 2a). As shown in Fig. 2b, nuclear and cytoplasmic mRNA grading assay was used to distinguish the subcellular localization of circ_0004913 in SMCC-7721 and Huh-7 cells, and circ_0006168 was mainly distributed in the cytoplasm. In order to study the potential function of circ_0004913 in regulating the process of $\mathrm{HCC}$, we transduced the stably expressed circ_0004913 into Huh-7 and SMCC-7721 cells by using the lentivirus pHBLV-CMV-circ_0004913. After stable expression, the expression level of circ_0004913 increased significantly, while the expression level of TEX2 mRNA did not change significantly (Fig. 2c). Compared with the NC group, overexpression of circ_0004913 decreased the proliferation rate of SMCC-7721 and Huh-7 cells (Fig. 2d and e). To further find out the underlying mechanisms, flow cytometry was performed to analyze the correlation between cell cycle changes and circ_0004913 expression. As indicated in Fig. 2f, circ_0004417 overexpression could result in a significant increase in the percentage of HCC cells in the G1 phase. Therefore, overexpression of circ_0004913 inhibits malignant behavior by attenuating the proliferation of HCC cells.

\section{Circ_0004913 acted as a ceRNA to sponge miR-1290}

Previous literature has shown that circRNA may serve as ceRNA to play the role of sponge miRNA, thereby reducing its inhibitory effect on targeted mRNA expression [17]. To understand the role of circRNA-0004913, 


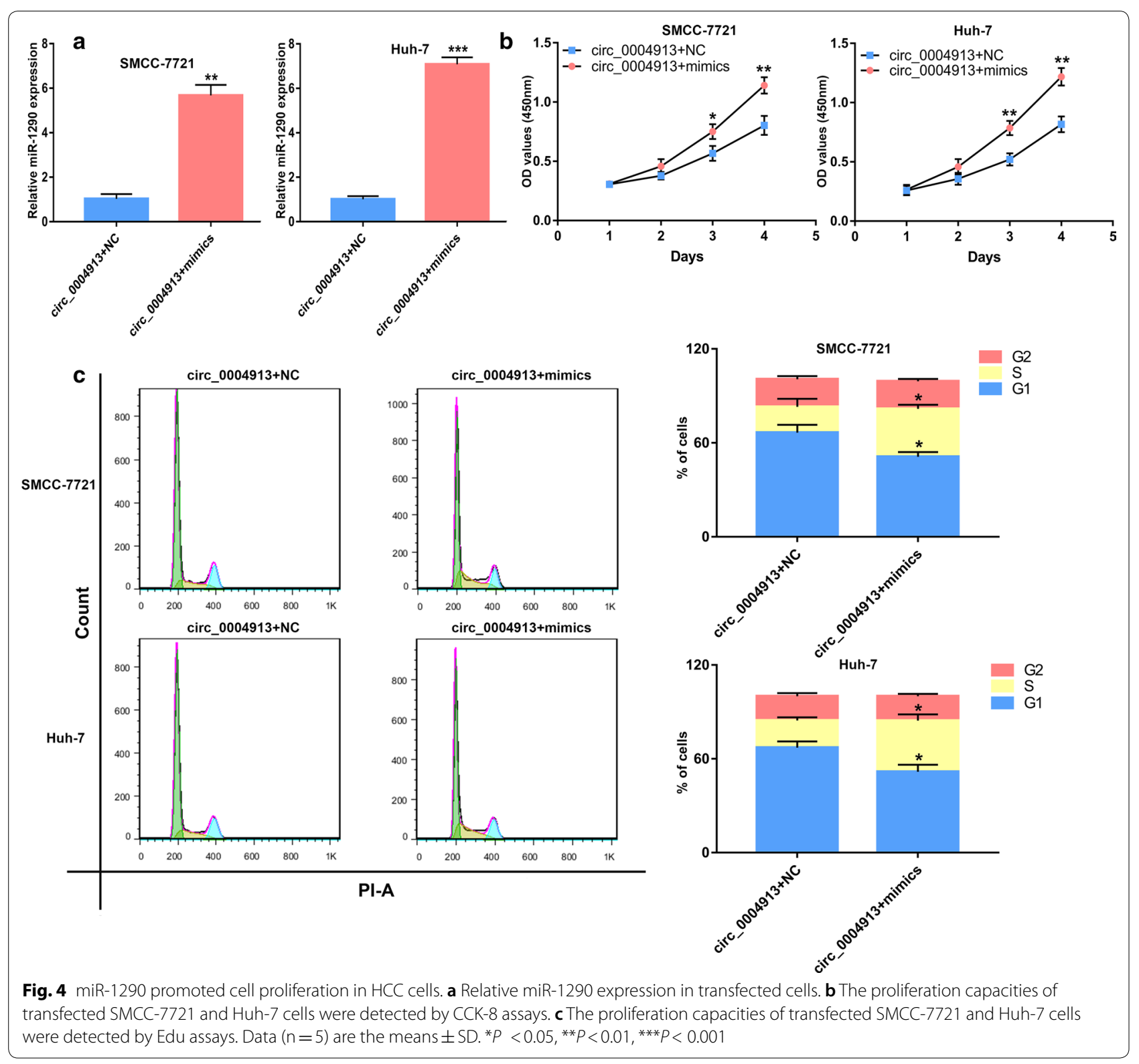

we first searched for potential targeted miRNAs in the Circular RNA Interactome and circRNA Bank databases through bioinformatics. MiR-1290 may combine with circ_0004913 in the cytoplasm (Fig. 3a). Co-transfection of miR-1290 mimics and the plasmid circ_0004913 wt could significantly reduce the luciferase activity in HEK293T cells (Fig. 3b). Then, RIP assay showed that circ_0004913 and miR-1290 were remarkably enriched in the Ago2 immunoprecipitation compared with the IgG immunoprecipitation in HEK293T cells (Fig. 3c). In addition, circ_0004913 overexpression significantly reduced the relative levels of miR-1290 in SMCC-7721 and Huh-7 cells (Fig. 3d). Compared with the adjacent liver tissue, the expression level of miR-1290 in HCC tissues were significantly increased, and it was negatively correlated with the expression level of circ_0004913 (Fig. 3e and f). Such data suggested that circ_0004913 may sponge miR-1290 to exert its biological function.

\section{MiR-1290 enhanced the proliferation of HCC cells}

To investigate the role of $\mathrm{miR}-1290$ in regulating the progression of HCC, we transfected miR-1290 mimics in SMCC-7721 and Huh-7 cells that with stably circ_0004913 overexpression (Fig. 4a). Compared with the control group, overexpression of miR-1290 significantly enhanced the proliferation of SMCC-7721 
a

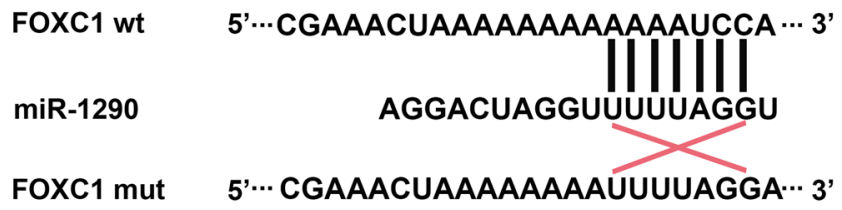

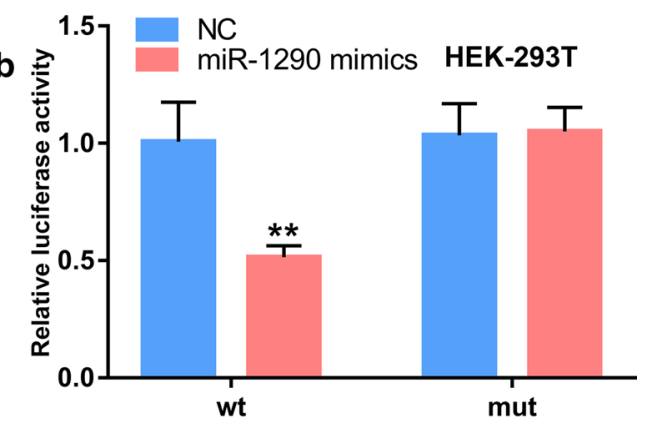
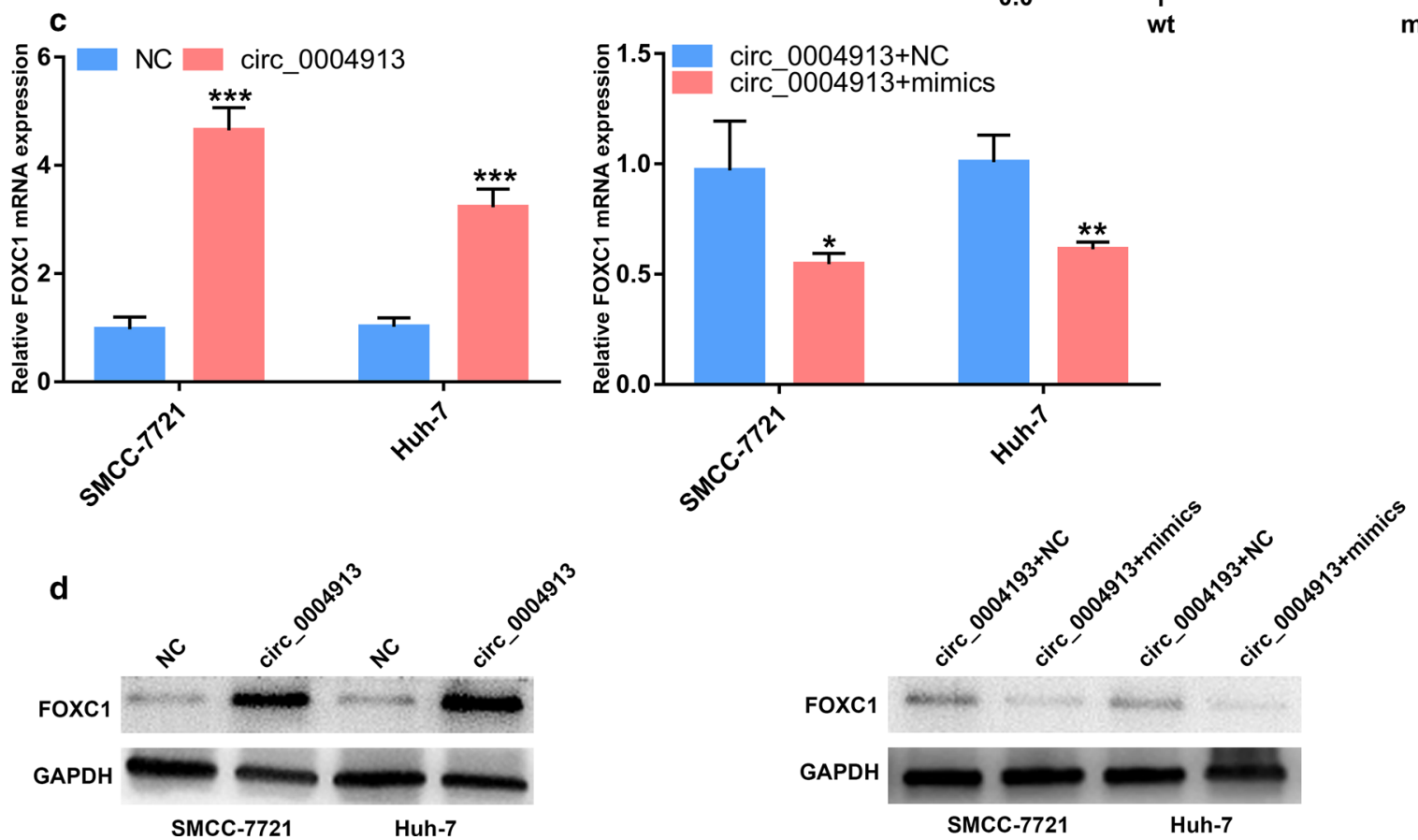

Fig. 5 circ_0004913 regulated miR-1290/FOXC1 axis. a Bioinformatics tools indicated the complementary binding sites of miR-1290 with FOXC1. b Luciferase reporter assay showed the targeting of miR-1290 with FOXC1 in HEK293T. c qRT-PCR showed the FOXC1 mRNA expression in transfected SMCC-7721 and Huh-7 cells. $\mathbf{d}$ Western blot showed the FOXC1 protein expression in transfected cells. Data $(n=5)$ are the means \pm SD. ${ }^{*} P<0.05,{ }^{*} P<0.01,{ }^{* *} P<0.001$

and Huh-7 cells and reversed the inhibitory effect of circ_0004913 on cell proliferation (Fig. 4b). Similarly, miR-1290 mimics promoted the transformation of cells from $\mathrm{G1}$ phase to $\mathrm{S}$ phase, and accelerated cell division (Fig. 4c). Such data indicated that miR-1290 could reverse the role of circ_0004913 in cells and enhanced the malignant behaviors of $\mathrm{HCC}$ cells.

\section{FOXC1 may be a potential target of miR-1290}

Next, using miRDB and TargetScan to predict the potential target genes of miR-1290 through bioinformatics. Since miR-1290 enhanced the malignant behavior of HCC cells, we searched for putative target genes with tumor suppressor function. Among the potential target genes of miR-1290, FOXC1 gene was a tumor suppressor and was used as the object of this study (Fig. 5a). Further luciferase assays showed that transfection with
miRNA-1290 mimics but not mutated significantly reduced FOXC1-mediated luciferase activity in HEB293T cells (Fig. 5b). After overexpressing circ_0004913, the mRNA and protein levels of FOXC1 in cells increased significantly. After transfection with miR-1290 mimics again, the expression level of FOXC1 decreased significantly (Fig. 5c and d).

\section{Circ_0004913 suppressed the proliferation of HCC cells in vivo}

Lastly, to further confirm our conclusion in vivo, we performed xenograft tumor model with SMCC7721 cells. BALB/c nude mice were monitored every three days, and euthanized in 5 weeks. By measuring tumor volume, we found that tumors from SMCC7721 circ_0004913 cells grew slower than those from the control group (Fig. 6a, b). Similarly, tumor weight 


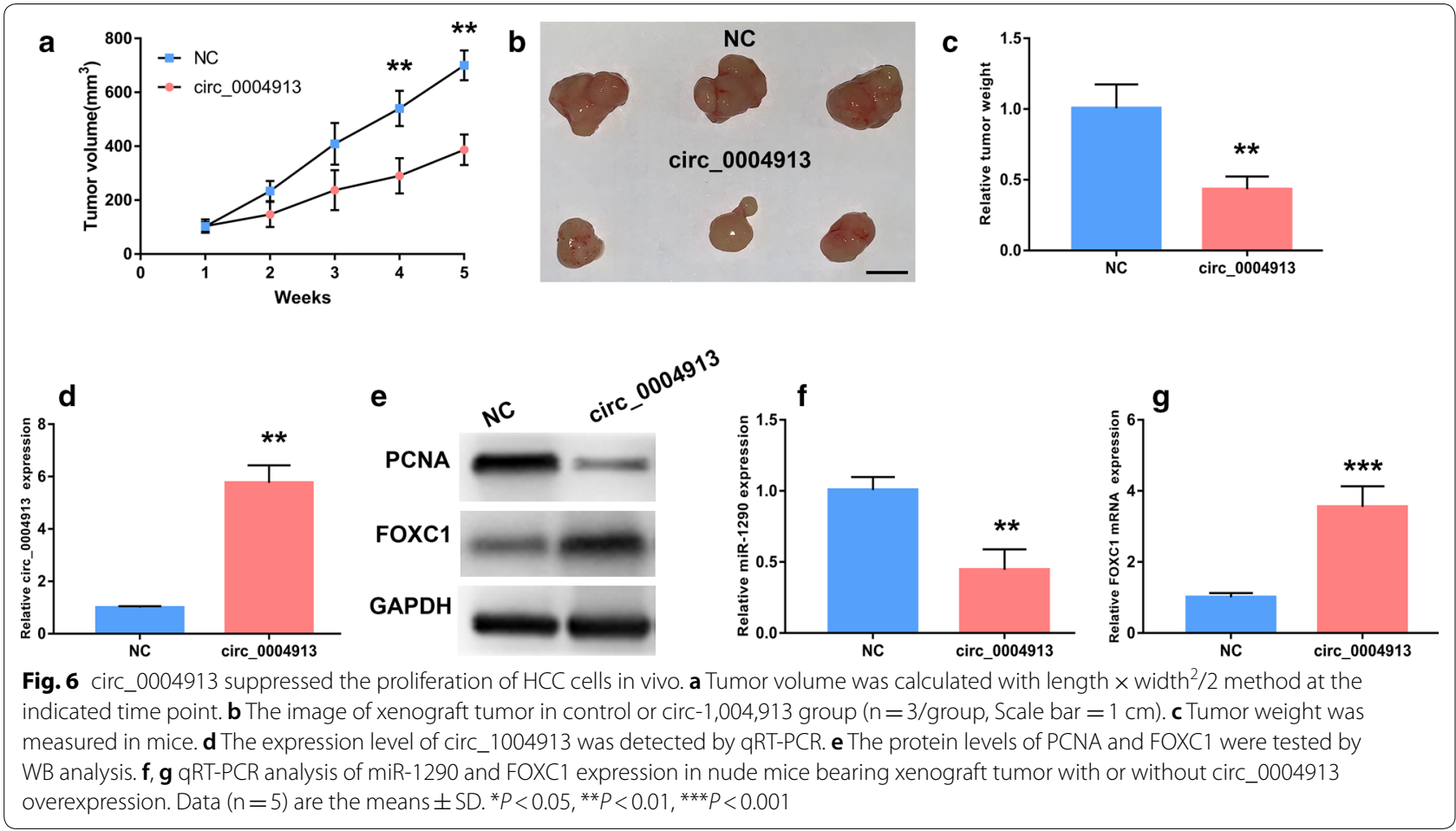

was significantly decreased in the SMCC-7721 cells inoculated with circ_0004913 overexpression (Fig. 6c). Moreover, we detected the protein levels of PCNA in xenograft tumors and found that circ_0004913 remarkably suppressed PCNA expression, indicating the inhibitory effect of circ_0004913 on HCC proliferation in vivo (Fig. 6d). Importantly, qRT-PCR results showed that circ_0004913 and FOXC1 expression were successfully increased, while miR-1290 expression was decreased in circ_0004913 overexpressed group in comparison to control group (Fig. 6e, f).Taken together, these results suggest that circ_0004913 inhibits tumor growth in vivo, consistent with our data in vitro.

\section{Discussion}

Our research indicated that circ_0004913 acted as a tumor suppressor through the miR-1290/FOXC1 axis to inhibit HCC cancer cell proliferation, suggesting that circ_0004913 may be a potential biotherapeutic target for HCC. Our findings supported: (1) circ_0004913 was down-regulated in HCC tissues and HCC cell lines, which was positively associated with poor clinical pathological features and overall survival of HCC patients; (2) circ_0004913 could delay the cell cycle progression of HCC cell lines and decrease the rate of cell proliferation; (4) circ_0004913 was a miR-1290 sponge, and circ_0004913 overexpression could significantly inhibit cell growth; (5) circ_0004913 sponged miR-1290 to promote FOXC1 expression and was necessary to regulate cancer progression. Therefore, our study identified the previously unknown role of circ_0004913 in inhibiting the occurrence and development of HCC.

Circ_0004913 is derived from TEX2. In this study, circ_0004913 was down-regulated in HCC tissue samples and cell lines, which is consistent with the results from other institutions. Lower expression of circ_0004913 was significantly associated with high level of AFP, history of liver cirrhosis, larger tumors, and distant metastases. Previous studies have shown that circRNAs could play a crucial role in cell cycle progression and proliferation [18, 19]. After RNase $R$ treatment, circ_0004913 was still detected with a little degradation. We provided evidence that ectopic expression of circ_0004913 could delay the cycle progression of HCC cells. These results indicated that circ_0004913 was closely related to the malignant progression of HCC.

Although the mechanism through which circRNA regulates carcinogenesis and cancer progression has not yet been fully elucidated, the "circRNA-miRNA-mRNA" axis, also known as the "miRNA sponge", has shown its potentiality [20]. In our research, we confirmed that the content of circ_0004913 in the cytoplasm was significantly higher than that in the nucleus, which also 
provides a basis for circ_0004913 to sponge miRNAs [21]. Circ_0004913 may be a miR-1290 sponge. miR-1290 has been reported to show a high expression in the tissues and blood of various cancer patients [22-25]. For example, miR-1290 promote colorectal cancer cell proliferation by targeting INPP4B and accelerated the metastasis of oral squamous cell carcinoma by inhibiting CCNG2 expression [23, 26]. MiR-1290 was also highly expressed in the plasma of cancer patients, and has the potential to be a tumor marker and to guide the prognosis of patients [27-29]. We hypothesized that overexpression of circ_0004913 could significantly reduce the expression of miR-1290, thereby inhibiting the proliferation, migration and invasion of HCC cell lines. We confirmed the direct correlation between miR-1290 and circ_0004913 through dual luciferase assay and RIP assay. MiR-1290 was significantly upregulated in HCC cells and tissues, and its overexpression promoted cell proliferation and accelerated cell transformation from G0/G1 phase to $S$ phase. Therefore, an increase in circ_0004913 expression in HCC cells leads to a decrease in miR-1290 expression, thereby inhibiting proliferation and cell cycle progression. Our results provided evidence that miR-1290 sponge caused by circ_004913 drives HCC progression, and circ_0004913 is the upstream target of miR-1290.

Next, through bioinformatics and luciferase reporter gene analyses, we confirmed that the target gene of circ_004913/miR-1290 was FOXC1. A large number of latest data indicated that FOXC1 was involved in the development of cancers, including HCC, and FOXC1 played a inhibitory role in the proliferation, migration, invasion and metastasis of HCC cells [25, 26]. It has been shown that overexpression of FOXC1 in HCC cells inhibits epithelial to mesenchymal transformation, migration and invasion in vitro [27]. We additionally revealed that circ_0004913/miR-1290 regulated FOXC1, as part of the sponge mechanism. Increasing circ_0004913 expression promoted FOXC1 expression, while increasing miR-1290 expression inhibited FOXC1 expression. To our knowledge, our study is the first to prove that circ_0004913 is involved in FOXC1 expression. These findings indicated that circ_0004913 protect FOXC1 from miR-1290-mediated degradation in a competitive endogenous RNAmediated manner.

We admitted that our research still has limitations. circRNA may rely on other mechanisms in the occurrence and development of HCC. The role of circ_0004913 in HCC remains to be explored. We still need to carry out experiments with larger sample size to evaluate the expression stability of circ_0004913 in peripheral blood of patients with liver cancer.
Table 1 Association between circ_0004913 expression and clinicopathologic features of patients with hepatocellular carcinoma

\begin{tabular}{|c|c|c|c|}
\hline \multirow[t]{2}{*}{ Variables } & \multicolumn{2}{|c|}{ circ_0004913 expression } & \multirow[t]{2}{*}{$P$ value } \\
\hline & High $n=25$ & Low $n=25$ & \\
\hline Age (years) & & & 0.571 \\
\hline$\leq 60$ & 12 & 14 & \\
\hline$>60$ & 13 & 11 & \\
\hline Gender & & & 0.774 \\
\hline Female & 11 & 10 & \\
\hline Male & 14 & 15 & \\
\hline HBs antigen & & & 0.248 \\
\hline Absent & 8 & 12 & \\
\hline Present & 17 & 13 & \\
\hline Liver cirrhosis & & & 0.771 \\
\hline With & 16 & 15 & \\
\hline Without & 9 & 10 & \\
\hline $\operatorname{AFP}(\mathrm{ng} / \mathrm{ml})$ & & & 0.556 \\
\hline$\leq 200$ & 10 & 8 & \\
\hline$>200$ & 15 & 17 & \\
\hline Tumor size & & & $0.009^{* *}$ \\
\hline$\leq 3 \mathrm{~cm}$ & 20 & 11 & \\
\hline$>3 \mathrm{~cm}$ & 5 & 14 & \\
\hline Vascular invasion & & & $0.047^{*}$ \\
\hline Absent & 15 & 8 & \\
\hline Present & 10 & 17 & \\
\hline TNM stage & & & $0.011^{*}$ \\
\hline$|-| \mid$ & 18 & 9 & \\
\hline III-IV & 7 & 16 & \\
\hline Edmondson grade & & & $0.024^{*}$ \\
\hline$|-| \mid$ & 16 & 8 & \\
\hline III-IV & 9 & 17 & \\
\hline
\end{tabular}

${ }^{*} P<0.05,{ }^{*} P<0.01$

\section{Conclusion}

Circ_0004913 was significantly down-regulated in HCC tissues and cell lines. The up-regulation of circ_0004913 significantly inhibited the proliferation and cycle progression of HCC cells. Circ_0004913 sponged miR-1290 to regulate FOXC1 expression. Therefore, circ_0004913 could be used as a promising prognostic biomarker and a therapeutic target for HCC patients.

\section{Abbreviations}

CircRNAs: Circular RNAs; HCC: Hepatocellular carcinoma; GEO: Gene Expression Omnibus database; qRT-PCR: Quantitative reverse transcription PCR; FOXC1: Forkhead box C1; TEX2: Testis expressed 2; HBV: Chronic hepatitis B virus; HCV: Hepatitis C virus; miRNAs: MicroRNAs; CCK8: Cell Counting Kit-8; Edu: 5-Ethynyl-2'-deoxyuridine; RIP: RNA immunoprecipitation. 


\section{Acknowledgements \\ None.}

\section{Authors' contributions}

Conception and design: YY, FQ. Development of methodology: SH. Acquisition of data: YS. Analysis and interpretation of data: ML. Writing, review, and revision of article: YY. All authors read and approved the final manuscript.

\section{Funding}

This work was supported by the Scientific development funding of Nanjing medical university (NMUB2018259).

\section{Availability of data and materials}

All data generated during this study are included in this published article.

\section{Ethics approval and consent to participate}

This study was authorized by the Ethics Committee of Huaian No.1 People's Hospital; all animal procedures were approved by the Animal Committee of Affiliated Huaian No.1 People's Hospital of Nanjing Medical University (IACUC-1906548).

\section{Consent for publication}

All authors approved publication of the manuscript.

\section{Competing interests}

The authors declare that they have no financial conflicts of interest.

\section{Author details}

${ }^{1}$ Department of Hepatobiliary Surgery, The Affiliated Huaian No.1 People's Hospital of Nanjing Medical University, 1 West Huanghe Road, Huai'an 223300, Jiangsu, People's Republic of China. ${ }^{2}$ Department of Ultrasonography, Lianshui People's Hospital Affiliated to kangda college of Nanjing Medical University, Huai'an 223300, Jiangsu, China. ${ }^{3}$ Department of Pathology, The Affiliated Huaian No.1 People's Hospital of Nanjing Medical University, Huai'an 223300, Jiangsu, China.

Received: 17 June 2020 Accepted: 26 August 2020

Published online: 03 September 2020

\section{References}

1. Bray F, et al. Global cancer statistics 2018: GLOBOCAN estimates of incidence and mortality worldwide for 36 cancers in 185 countries. CA Cancer J Clin. 2018;68(6):394-424.

2. Kulik L, El-Serag HB. Epidemiology and management of hepatocellular carcinoma. Gastroenterology. 2019;156(2):477-91.e1.

3. Singal AG, El-Serag HB. Hepatocellular carcinoma from epidemiology to prevention: translating knowledge into practice. Clin Gastroenterol Hepatol. 2015;13(12):2140-51.

4. Grandhi MS, et al. Hepatocellular carcinoma: from diagnosis to treatment. Surg Oncol. 2016;25(2):74-85.

5. Nishida N, Kudo M. Oncogenic signal and tumor microenvironment in hepatocellular carcinoma. Oncology. 2017;93(Suppl 1):160-4.

6. Greene J, et al. Circular RNAs: biogenesis, function and role in human diseases. Front Mol Biosci. 2017:4:38.

7. Tang Q, Hann SS. Biological roles and mechanisms of circular RNA in human cancers. Onco Targets Ther. 2020;13:2067-92.

8. Lu C, et al. Knockdown of circular RNA circ-FARSA restricts colorectal cancer cell growth through regulation of miR-330-5p/LASP1 axis. Arch Biochem Biophys. 2020;689:108434.

9. Dong L, Qu F. CircUBAP2 promotes SEMA6D expression to enhance the cisplatin resistance in osteosarcoma through sponging miR-506-3p by activating Wnt/ß-catenin signaling pathway. J Mol Histol. 2020;51(4):329-40.

10. Chen LL. The biogenesis and emerging roles of circular RNAs. Nat Rev Mol Cell Biol. 2016;17(4):205-11.

11. Li R, Luo S, Zhang D. Circular RNA hsa_circ_0054537 sponges miR$130 \mathrm{a}-3 \mathrm{p}$ to promote the progression of renal cell carcinoma through regulating cMet pathway. Gene. 2020;754:144811.

12. Chen $\mathrm{L}$, et al. Circ_0008532 promotes bladder cancer progression by regulation of themiR-155-5p/miR-330-5p/MTGR1 axis. J Exp Clin Cancer Res. 2020;39(1):94.

13. Aghaee-Bakhtiari SH. Online databases and circular RNAs. Adv Exp Med Biol. 2018;1087:35-8.

14. Han D, et al. Circular RNA circMTO1 acts as the sponge of microRNA-9 to suppress hepatocellular carcinoma progression. Hepatology. 2017:66(4):1151-64.

15. Fu L, et al. Screening differential circular RNA expression profiles reveals hsa_circ_0004018 is associated with hepatocellular carcinoma. Oncotarget. 2017:8(35):58405-16

16. Xiong DD, et al. A circRNA-miRNA-mRNA network identification for exploring underlying pathogenesis and therapy strategy of hepatocellular carcinoma. J TransI Med. 2018;16(1):220.

17. He Y, et al. CircZNF609 enhances hepatocellular carcinoma cell proliferation, metastasis, and stemness by activating the Hedgehog pathway through the regulation of miR-15a-5p/15b-5p and GLI2 expressions. Cell Death Dis. 2020;11(5):358.

18. Wang Y, et al. Circular RNA hsa_circ_0003141 promotes tumorigenesis of hepatocellular carcinoma via a miR-1827/UBAP2 axis. Aging. 2020;12:9793-806.

19. Wang M, Yu F, Li P. Circular RNAs: characteristics, function and clinical significance in hepatocellular carcinoma. Cancers. 2018;10(8):258.

20. Kulcheski FR, Christoff AP, Margis R. Circular RNAs are miRNA sponges and can be used as a new class of biomarker. J Biotechnol. 2016;238:42-51.

21. Huang J, et al. Exosome-mediated transfer of miR-1290 promotes cell proliferation and invasion in gastric cancer via NKD1. Acta Biochim Biophys Sin. 2019;51(9):900-7.

22. Qin WJ, et al. MiR-1290 targets CCNG2 to promote the metastasis of oral squamous cell carcinoma. Eur Rev Med Pharmacol Sci. 2019;23(23):10332-42.

23. Sun Y, et al. miR-1290 promotes proliferation and suppresses apoptosis in acute myeloid leukemia by targeting FOXG1/SOCS3. J Biol Regul Homeost Agents. 2019;33(6):1703-13.

24. Wei J, et al. Serum miR-1290 and miR-1246 as potential diagnostic biomarkers of human pancreatic cancer. J Cancer. 2020;11(6):1325-33.

25. Xia L, et al. Overexpression of forkhead box $\mathrm{C} 1$ promotes tumor metastasis and indicates poor prognosis in hepatocellular carcinoma. Hepatology. 2013;57(2):610-24.

26. $\mathrm{Xu} \mathrm{Z}$, et al. C/EBPa regulates FOXC1 to modulate tumor growth by interacting with PPARY in hepatocellular carcinoma. Curr Cancer Drug Targets. 2020;20(1):59-66.

27. Xu ZY, et al. FOXC1 contributes to microvascular invasion in primary hepatocellular carcinoma via regulating epithelial-mesenchymal transition. Int J Biol Sci. 2012;8(8):1130-41.

28. Liu X, et al. Circulating miR-1290 and miR-320d as novel diagnostic biomarkers of human colorectal cancer. J Cancer. 2019;10(1):43-50.

29. Ng PC, et al. Plasma miR-1290 Is a novel and specific biomarker for early diagnosis of necrotizing enterocolitis-biomarker discovery with prospective cohort evaluation. J Pediatr. 2019;205(83-90):e10.

\section{Publisher's Note}

Springer Nature remains neutral with regard to jurisdictional claims in published maps and institutional affiliations. 